\title{
Dissociation of sensory-attentional from motor-intentional neglect
}

\author{
Duk L Na, John C Adair, David J G Williamson, Ronald L Schwartz, Barbara Haws, \\ Kenneth M Heilman
}

\begin{abstract}
Objectives-Spatial neglect may result from disruption of sensory-attentional systems that spatially allocate perceptual resources and the motor-intentional systems that direct exploration and action. Previous studies have suggested that the line bisection task is more sensitive to sensory-attentional disorders and the cancellation task to motor-intentional disorders. A new technique was developed that allows the dissociation of sensoryattentional and motor-intentional deficits in both tasks and thereby allows comparison of these tasks.

Methods-Ten patients with right hemispheric injury and hemispatial neglect performed line bisection and cancellation tasks while viewing stimuli on closed circuit TV. Direct view of the exploring hand and the target was precluded; the TV monitor guided performance. The direct condition made the direction of hand movement on the table (workspace) congruent with that on the monitor. Inverting the camera produced the indirect condition wherein the lateral movement in the workspace occurred in the opposite direction on the monitor.
\end{abstract}

Results-On the cancellation task, five patients marked targets in the right workspace in the direct condition but the left workspace in the indirect condition, indicating sensory-attentional neglect. However, four other patients cancelled targets only in the right workspace in both conditions, failing to explore the left workspace, suggesting motor-intentional neglect. A patient who performed ambiguously may have elements of both types of neglect. Only two out of five patients designated as sensory-attentional in cancellation tasks showed sensory neglect on line bisection. The other three patients, as well as patients defined as motor-intentional by cancellation performance, exhibited motor-intentional neglect on line bisection.

Conclusion-The designation of sensoryattentional versus motor-intentional neglect therefore, in part, depends on task specific demands.

\section{(F Neurol Neurosurg Psychiatry 1998;64:331-338)}

Keywords: neglect; inattention; sensory neglect; motor neglect; hemispatial neglect
Hemispatial or unilateral spatial neglect refers to a patient's failure to report, respond, or orient to stimuli presented to the side of space opposite a brain lesion despite intact elementary sensory and motor function. ${ }^{1}$ Line bisection and target cancellation (cancellation) are two tasks often used to test for spatial neglect. However, rightward bisection error and the omission of targets from the left side of an array correlate poorly. Some patients with hemispatial neglect place their mark far to the right of centre but cancel all targets, whereas others show the opposite pattern of performance. ${ }^{2}$ Hence, the two tasks may place qualitatively different demands on the patient and may be assessing different systems that contribute to spatial cognition.

Many theories have been posited to explain the neglect syndrome. Most contemporary accounts attribute neglect to sensoryattentional, motor-intentional, or representational deficits. ${ }^{134}$ Coslett et al, ${ }^{5}$ Halligan and Marshall, ${ }^{6}$ Bisiach et al, ${ }^{7}$ Tegner and Levander, ${ }^{8}$ and Harvey et al described different methods that dissociated intentional from attentional neglect. In all of these studies, functional dissociation seemed to relate to anatomical dissociation whereby frontal lesions were associated with motor-intentional neglect and posterior lesions produced sensory-attentional neglect.

Similar dissociations were shown in a patient who sustained sequential strokes in different parts of the right hemisphere. ${ }^{10}$ The initial injury in the frontal lobe resulted in hemispatial neglect on a manual spatial exploration task and a second stroke in the ipsilateral parietal region produced sensory extinction. Binder et $a l^{11}$ reported that the combination of abnormal cancellation performance with normal line bisection performance was consistently associated with lesions of the frontal lobe or basal ganglia. As anterior lesions had been associated with motor-intentional and exploratory deficits, they asserted that the cancellation task was more sensitive in detecting the motorintentional type of neglect and the line bisection task was more sensitive to the sensory-attentional form. However, by contrast with the other studies that dissociated attention and intention, ${ }^{5-9}$ the report of Binder et al did not directly decouple attentional from intentional influences on the line bisection and cancellation tasks.

Although the techniques described above ${ }^{5-9}$ dissociate sensory-attentional and motorintentional influences, these methods cannot be used to directly compare line bisection per- 


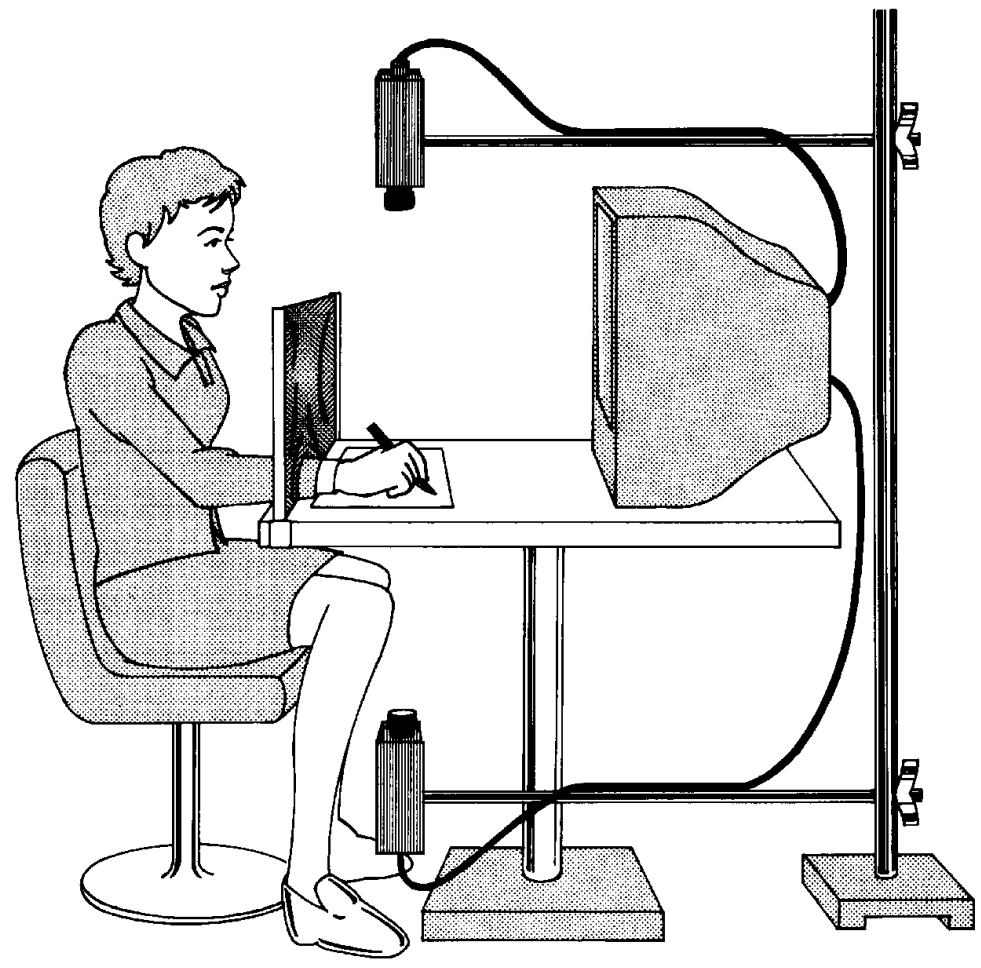

Figure 1 Position of subject in relation to table (workspace) and monitor (perceptual space).

formance with cancellation performance. Milner and Harvey ${ }^{12}$ state the critical question explicitly: do the different methods tap into the same taxonomic distinction between subtypes of neglect? Because techniques used in prior studies could not be used to dissociate attentional and intentional disorders in both line bisection and cancellation tasks, we developed a novel apparatus that not only decouples the direction of visual attention from the direction of hand movement but also allows application to both line bisection and cancellation tasks.

\section{Methods}

SUBJECTS

A group of 10 right handed patients with right hemispheric ischaemic stroke participated in our research. There were eight men and two women with a mean age of 64.6 (SD 9, range 42-72 years). All patients showed elements of left hemispatial neglect, demonstrating rightward bias in either line bisection or cancellation. Most patients were examined during the initial period in hospital (range 7-30 days). Only three patients were studied in a more

Table 1 Clinical details of patient group

\begin{tabular}{|c|c|c|c|c|c|c|c|}
\hline Case & $\begin{array}{l}\text { Time since } \\
\text { onset (days) }\end{array}$ & Hemiparesis & $\begin{array}{l}\text { Hemisensory } \\
\text { loss }\end{array}$ & $\begin{array}{l}\text { Tactile } \\
\text { extinction }\end{array}$ & $\begin{array}{l}\text { Visual } \\
\text { extinction }\end{array}$ & Hemianopia & Lesion \\
\hline 1 & 7 & + & + & + & + & - & $\mathrm{TP} / \mathrm{SC}$ \\
\hline 2 & 7 & + & + & + & + & + & $\mathrm{TP}$ \\
\hline 3 & $\sim 270$ & + & - & - & - & - & FP \\
\hline 4 & 13 & + & - & - & - & - & FT/SC \\
\hline 5 & $\sim 180$ & + & - & + & + & - & $\mathrm{P}$ \\
\hline 6 & $\sim 240$ & + & + & + & - & - & $\mathrm{F} / \mathrm{SC}$ \\
\hline 7 & 20 & + & - & - & - & - & $\mathrm{F}$ \\
\hline 8 & 60 & + & - & - & - & - & $\mathrm{FP} / \mathrm{SC}$ \\
\hline 9 & 30 & + & + & + & + & - & $\mathrm{P}$ \\
\hline 10 & 9 & + & + & + & + & - & SC \\
\hline
\end{tabular}

$\mathrm{F}=$ frontal; $\mathrm{T}=$ temporal; $\mathrm{P}=$ parietal; $\mathrm{SC}=$ subcortical chronic phase during or after rehabilitation (range 2-9 months after onset).

APPARATUS

Patients performed line bisection and cancellation tasks while viewing stimuli on closed circuit TV (fig 1). Manipulation of the video camera created two different conditions. In the direct condition, the left side of the paper was on the left of the screen and the right side of the paper was on the right side of the screen. Movements on the table (workspace) were thereby congruent with those displayed on the monitor (perceptual space). Inverting the camera perspective by $180^{\circ}$ created the indirect condition. In the indirect condition, the left side of the paper projected to the right side of the monitor and vice versa. Consequently, leftward movements in the workspace appear as rightward movements in perceptual space and vice versa.

Patients sat at a clear Plexiglas table on which a 12 inch, black and white TV monitor was positioned parallel to their coronal plane at a distance of about $0.5 \mathrm{~m}$. A drape placed between the patient and the table precluded direct view of the target stimuli or their own hand but allowed an unobstructed view of the monitor. Thus the image on the screen guided task performance. The video camera was positioned above (direct condition) or below (indirect condition) the table. Using a carpentry level, the long axis of the video camera was made perpendicular to the surface of the table. The video camera (Panasonic WV-1550) was equipped with a standard TV lens that had a focal length of $12.5 \mathrm{~mm}$ and a maximum aperture of $\mathrm{F} / 1.3$. The distance between the video camera and the surface of the table (about 400 $\mathrm{mm}$ ) was adjusted such that dimensions depicted on the screen were roughly equal to dimensions on the stimulus paper. The camera distance resulted in projection of the border of the stimulus paper just beyond the margin of the screen. This precluded the use of any nonstimulus spatial cues.

In both the direct condition and the indirect condition, the same stimulus pages were used with the printed side facing up. Thus the indirect condition required back projection of the marker point and the hand's shadow through the stimulus page, resulting in a different image than the direct condition. However, ample illumination above the table and adjustment of the aperture of the lens produced clear targets (lines) and allowed satisfactory visual guidance during task performance.

\section{PROCEDURES}

The lines for the bisection task were $230 \mathrm{~mm}$ in length and $3 \mathrm{~mm}$ thick. Lines were centred on white A4 size paper $(278 \times 216 \mathrm{~mm})$. Deviations from objective midpoint were measured to the closest $\mathrm{mm}$; positive values denote rightward deviation and negative values denote leftward deviation.

We used a modified version of Albert's cancellation test. ${ }^{13}$ In this task, 40 black lines (25 $\mathrm{mm}$ length, $0.5 \mathrm{~mm}$ thick) of various orientations were dispersed in a random array on a 
Table 2 Comparison of line bisection and target cancellation, direct $v$ indirect conditions

\begin{tabular}{llllllll}
\hline & \multicolumn{3}{l}{ Bisection task } & & & \multicolumn{2}{l}{ Cancellation task } \\
\cline { 2 - 3 } \cline { 6 - 7 } Patient & Direct & Indirect & ATT/INT & & $\begin{array}{l}\text { Direct } \\
\text { left-right (\%) }\end{array}$ & $\begin{array}{l}\text { Indirect } \\
\text { left-right (\%) }\end{array}$ & ATT/INT \\
\hline 1 & +27.4 & -50.6 & ATT & & $4-76$ & $60-0$ & ATT \\
2 & +1.7 & +18.2 & INT & & $60-85$ & $95-36$ & ATT \\
3 & +5.3 & +13.7 & INT & & $98-100$ & $26-26$ & Ambiguous \\
4 & +14.9 & +58.3 & INT & & $0-33$ & $0-31$ & INT \\
5 & +3.0 & +5.6 & INT & & $96-100$ & $100-97$ & ATT \\
6 & +4.1 & +9.6 & INT & & $56-100$ & $78-61$ & ATT \\
7 & +13.8 & +19.7 & INT & & $0-46$ & $0-30$ & INT \\
8 & +20.2 & +23.4 & INT & & $47-99$ & $0-36$ & INT \\
9 & +25.3 & -10.9 & ATT & & $0-87$ & $74-16$ & ATT \\
10 & +25.1 & +46.3 & INT & & $0-91$ & $30-50$ & INT \\
\hline
\end{tabular}

ATT $=$ sensory attentional; INT $=$ motorintentional.

$278 \times 216 \mathrm{~mm}$ sheet of white paper. There were 18 lines on each side of the page and four lines in the centre that were not included in data analysis.

Ten line bisections and one to three cancellations were obtained in both the direct and indirect conditions. The number of cancellation tasks attempted had to be tailored to the individual patient's degree of cooperation. The direct condition always preceded the indirect condition to verify the persistence of left hemispatial neglect detected in free field screening tasks. The screen and the stimulus paper were aligned in the midsagittal plane of the subject's body. For cancellation tasks, the

A

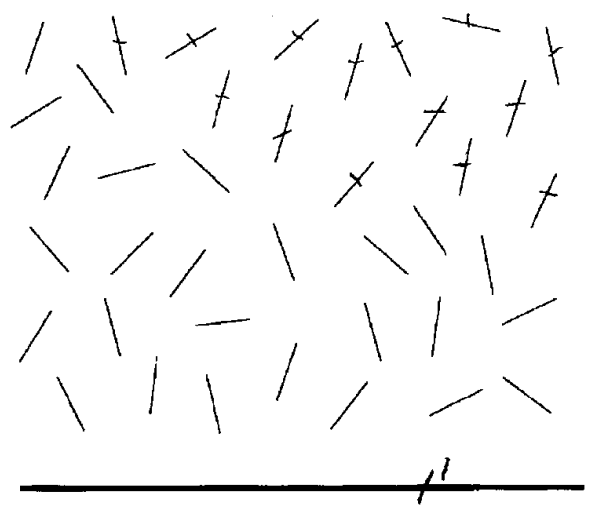

B

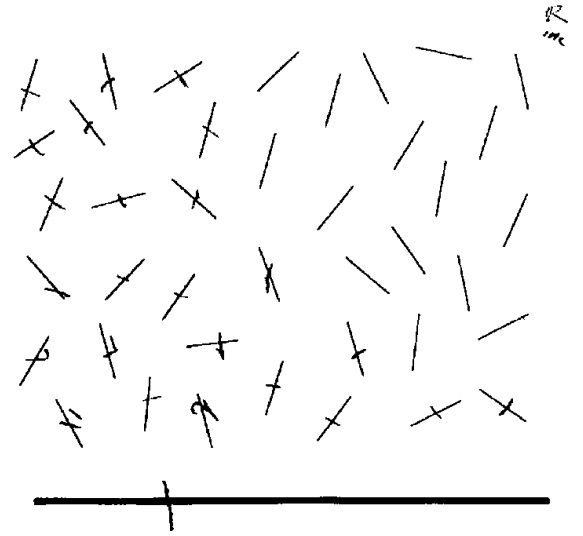

Figure 2 (A) In the direct condition, patient 1 misbisects the line to the right of true centre and cancels lines mainly on the right half of the array. (B) In the indirect condition, patient 1 misbisects the line to the left and cancels lines mainly on the left, a "mirror image" of performance in the direct condition. patient's hand was positioned in the centre of the array at the beginning of the trial. Before initiating the indirect condition, patients were reminded that their movements would not be in their "usual" expected direction but to persist despite frustration or disoriention. However, patients were not given a specific strategy for adjusting to the indirect condition. When patients moved their hand off the screen on cancellation tasks, their hand was returned to the starting point at the centre of the array. When patients paused during cancellation performance, they were asked if they had finished. Cancellation procedures were terminated if they indicated completion of the task or if they failed to locate any new targets for a period of five minutes. The duration of each procedure was about 30 minutes.

\section{Results}

All patients showed hemiparesis of differing grades of severity and half the patients also showed hemihypaesthesia (table 1). None of the patients showed a complete homonymous hemianopia, although one patient (2) had a quadrantic visual field deficit. Extinction to double simultaneous visual stimulation was present in five of 10 patients: extinction to tactile stimuli was present in these five patients as well as in an additional patient (6).

Most patients completed the task without difficulty. Table 2 shows that in the indirect condition, four patients had trouble maintaining their hand location in the workspace during the cancellation task. Eight of 10 patients showed some perseverative behaviour on the cancellation task whereby they reiteratively marked targets that they had previously cancelled. Among this group, six perseverated in both direct and indirect conditions whereas two other patients perseverated only in the indirect condition.

\section{LINE BISECTION}

In the direct condition, the mean bisection error was rightward for all patients. Although the degree of bisection error varied widely, all 10 patients exceeded the $95 \%$ confidence interval established with normal subjects $(-0.98 \mathrm{~mm}$ to $0.55 \mathrm{~mm})$. A rightward bias was also found on free field bisections during clinical evaluation, suggesting that the demands on perceptual processes during direct line bisection as guided by the monitor did not exceed the demands imposed by the traditional, free field line bisection task.

In the indirect condition, deviations on the monitor are opposite in direction to those on the actual line. Therefore, in this condition, rightward deviations on the actual line are leftward on the monitor and vice versa. Rightward deviations on the actual line would suggest a failure to move leftward (motor-intentional defict), whereas leftward deviations on the actual line would suggest defective perceptual processing of the left side of the monitor (sensory-attentional deficit). On the line bisection task, most patients (eight of 10) showed rightward deviation on the actual line (leftward on the monitor), supporting a 
A

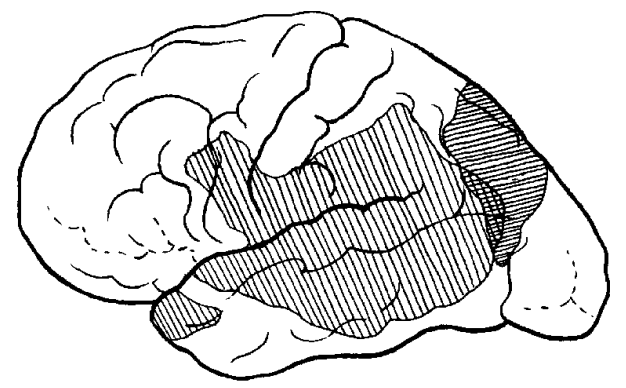

B

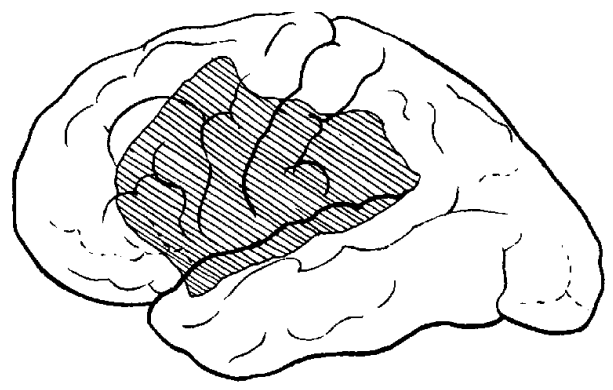

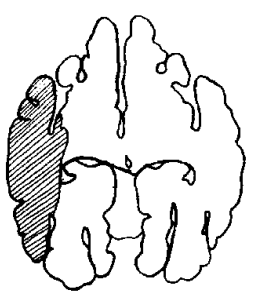
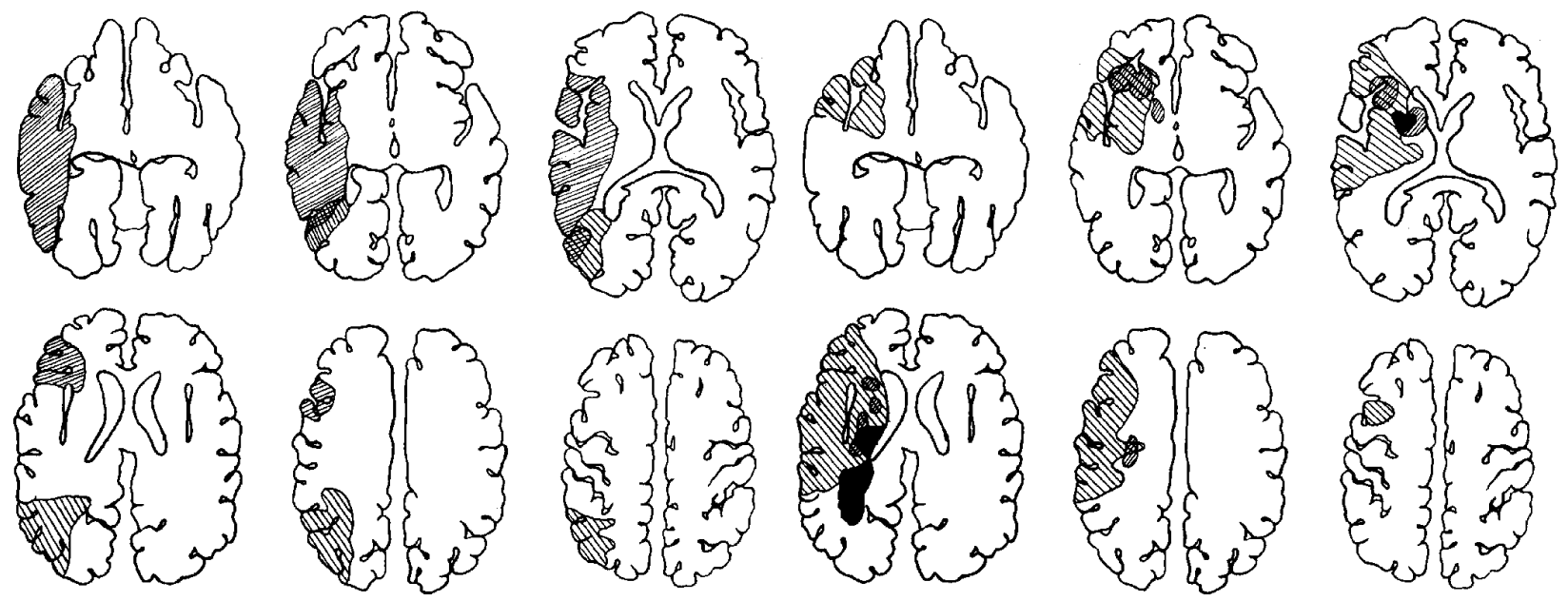

Figure 3 (A) Templates adapted from Damasio and Damasio ${ }^{14}$ showing the distribution of injury in two patients with attentional neglect on both line bisection and cancellation tasks. The lesions primarily involve structures posterior to the central sulcus. (B) Templates demonstrating the distribution of injury in two patients with intentional neglect on both line bisection and cancellation tasks. Although widespread, the lesion impacts more cortex anterior to the central sulcus compared with $(A)$.

motor-intentional defect. The other two patients erred to the left on the actual line (rightward on the monitor). This indicated that their performance was determined by visualperceptual information regardless of the space in which their hand was moving, a result consistent with primarily a sensory-attentional neglect. There was no clear relation between the clinical features listed in table 1 and performance pattern on the line bisection task.

\section{CANCELLATION}

In the direct condition, all patients showed some degree of impairment cancelling targets in left space. Performance varied widely, from complete failure to cancel any target in left space to omission of a single left sided target. Additionally, five patients also failed to find a normal number of targets in the right half of space. However, all patients cancelled more targets on the right side of the array than the left. Again, this pattern of performance was essentially identical to performance on free field cancellation tasks found during clinical evaluation.

In the indirect condition, five patients failed to cancel, in the actual workspace, more targets on the right side of the sheet than on the left. On the monitor, this would be a failure to cancel more targets on the left. In several instances, the inversion of cancellation performance was striking, producing a "mirror image" of the cancellation pattern obtained under the direct condition (fig 2). Therefore, on this cancellation task, five out of 10 failed to cancel targets based on their position in perceptual space (the left half of the monitor's image), a performance indicating primarily a sensory-attentional form of neglect. With one exception these five patients correspond to those patients in table 1 who showed either visual or tactile extinction. In the indirect task, another group of four patients failed to cancel more targets on the left, which would correspond to the right side of the monitor. Therefore, those patients seemed to exhibit deficient exploration toward left workspace, a result consistent with motor-intentional neglect.

One patient performed ambiguously, locating an equal number of targets in both hemispaces.

COMPARISON BETWEEN TASKS

Performance on these tasks defined three distinct groups of patients: two patients showed sensory-attentional neglect in both line bisection and cancellation tasks (A-A group), four patients showed motor-intentional neglect in both tasks (I-I group), and three patients exhibited motor-intentional neglect on the line bisection task and sensory-attentional neglect on the cancellation task (I-A group). The remaining patient's performance indicated a motor-intentional deficit on line bisection but cancellation performance could not be 
Table 3 Dissociation of attentional and intentional neglect types (anatomical correlation from combined studies)

\begin{tabular}{|c|c|c|c|}
\hline Source & Neglect type & Patient No & Lesion distribution \\
\hline \multirow[t]{7}{*}{ Bisiach et al } & \multirow[t]{5}{*}{ Sensory attentional } & 1 & FP \\
\hline & & 2 & $\mathrm{PO}$ \\
\hline & & 3 & $\mathrm{P}$ \\
\hline & & 4 & FP \\
\hline & & 9 & $\mathrm{PO}$ \\
\hline & \multirow[t]{2}{*}{ Motor intentional } & 8 & SC \\
\hline & & 10 & FP \\
\hline \multirow[t]{4}{*}{ Coslett $e t a \bar{P}$} & \multirow[t]{2}{*}{ Sensory attentional } & 3 & TPO \\
\hline & & 4 & FTP \\
\hline & \multirow[t]{2}{*}{ Motor intentional } & 1 & $\mathrm{FP} / \mathrm{SC}$ \\
\hline & & 2 & FT \\
\hline \multirow[t]{13}{*}{ Tegner and Levander ${ }^{8}$} & \multirow[t]{10}{*}{ Sensory attentional } & 1 & $\mathrm{TP}$ \\
\hline & & 4 & TP \\
\hline & & 5 & $\mathrm{O}$ \\
\hline & & 6 & $\mathrm{TP} / \mathrm{SC}$ \\
\hline & & 7 & 0 \\
\hline & & 11 & $\mathrm{P} / \mathrm{SC}$ \\
\hline & & 14 & $\mathrm{TP}$ \\
\hline & & 15 & $\mathrm{TP}$ \\
\hline & & 16 & 0 \\
\hline & & 17 & $\mathrm{P}$ \\
\hline & \multirow[t]{3}{*}{ Motor intentional } & 2 & FTP \\
\hline & & 8 & FTP \\
\hline & & 9 & $\mathrm{FP}$ \\
\hline \multirow{6}{*}{ Present study } & \multirow[t]{2}{*}{ Sensory attentional } & 1 & $\mathrm{TP} / \mathrm{SC}$ \\
\hline & & 9 & FP \\
\hline & \multirow{4}{*}{ Motor intentional } & 4 & $\mathrm{FT} / \mathrm{SC}$ \\
\hline & & 7 & F \\
\hline & & 8 & FP \\
\hline & & 10 & SC \\
\hline
\end{tabular}

$\mathrm{F}=$ frontal $; \mathrm{T}=$ temporal $; \mathrm{P}=$ parietal $\mathrm{O}=$ occipital $\mathrm{SC}=$ subcortical.

unambiguously classified. Overall, on the line bisection task, eight of 10 patients showed motor-intentional neglect and two showed sensory-attentional neglect. By contrast, on the cancellation test, five patients showed sensoryattentional neglect and four showed motorintentional neglect.

CLINICAL ANATOMICAL CORRELATION

All patients underwent either brain CT (three patients) or MRI (seven patients) during their stay in hospital. The lesions identified on axial studies were traced on oblique templates ${ }^{14}$ by a neurologist who was unaware of the patients' performance on the tasks described above. The templates were also used to reconstruct graphically the lesion distribution over the lateral surface of the hemisphere.

Templates from the A-A group were superimposed (fig 3A). The main area of involvement in this group includes the parietal lobe. Templates from the I-I group were likewise superimposed (fig 3B). The common anatomical distribution of the lesions was much more widespread, although anterior and subcortical structures appeared overrepresented. The three patients in the I-A group also had predominantly anterior lesions.

If we designate anterior lesions as those confined to the frontal lobe or basal ganglia and posterior lesions are defined as those involving the temporal, parietal, or occipital lobes, then only two of 10 patients had brain injury restricted to either of the two regions. The patient with the posterior lesion showed sensory-attentional neglect under both tasks and the patient with the anterior lesion showed motor-intentional neglect only for line bisection.

In the remaining eight patients, the predominant lesion location was anterior in four and posterior in four. Of eight patients with motorintentional neglect during the line bisection task, five showed predominantly anterior lesions. The two patients showing sensoryattentional neglect during the bisection task had predominantly posterior brain injury. Of five patients with sensory-attentional neglect on the cancellation task, three patients had lesions with posterior predominance. The location of lesions in four patients with motorintentional neglect on the cancellation task were predominantly anterior in three and posterior in one.

\section{Discussion}

The video apparatus described allows us to fractionate spatial neglect into sensoryattentional or motor-intentional subtypes. Although the dichotomy between attentional and intentional influences is probably not absolute, such techniques facilitate a rational nosology of neglect derived from theoretical principles. The distinction between different forms of neglect behaviour provides a more refined understanding of the specific deficit behind a given patient's defective interaction with stimuli from space opposite a brain lesion. Although research into neglect treatment is only at a preliminary stage, this distinction may be crucial to the type of rehabilitation measures chosen for a patient with neglect. ${ }^{15}$

In addition to directed attention and intention, some authors attribute left unilateral spatial neglect to destruction of the mental representation of left space. ${ }^{4}{ }^{16}$ Patients who do not have a representation of left space should fail to attend to stimuli in left space and should also fail to act in or toward left space. Following a strict interpretation of the "representational hypothesis," the fractionation of neglect into attentional and intentional subtypes is difficult to reconcile with a defective representation of left space. Patients with sensory-attentional type of neglect attend primarily to the right side of space but explore to the left. Similarly, patients with motorintentional neglect primarily explore the right workspace but this corresponds, under the indirect condition, to the left perceptual space. The indirect condition would therefore impose insurmountable demands on the patient with "representational" neglect; such a patient should fail to cancel targets in both right and left space and err to both sides of the midline on the bisection task. Admittedly, it is possible that processing of spatial information invokes separate representations for sensory-perceptual space and motor-intentional space, damage to either of which might produce the performances found in the present study.

The present study corroborates the findings from the modified line bisection task of Halligan and Marshall, ${ }^{6}$ Bisiach et al, ${ }^{7}$ Coslett et $a l,{ }^{5}$ and the cancellation task of Tegner and Levander. ${ }^{8}$ In addition, our device demonstrates several technical advantages over previously described methods.

For example, a potential drawback of Halligan and Marshall's computer based line bisection paradigm includes the fact that the 
"gain" setting on the manual control might produce an amplification effect in which magnitude of the displacement of the cursor exceeds the magnitude of displacement of the "mouse" by some constant factor. Such an influence might minimise the contribution of premotor factors to abnormal line bisection performance. The technique of Coslett et al could dissociate the hemispace of action and visual feedback but not the direction of movement required to interact with the stimuli. Although the device of Bisiach et al also dissociates the direction of movement from the direction of attention, its design inherently limits its application to line bisection. Although Tegner and Levander reported a reversal of error direction with line bisection in one patient, the seam created by the intersection of reflective surfaces in their device provides an inescapable cue regarding the midpoint of lines, assuming they are centred on the stimulus paper within the viewing "window" of the apparatus. By contrast, our method readily separates the hemispace of action and direction of movement from the hemispace of visuoperceptual feedback and direction of attention for both line bisection and target cancellation tasks. In principle, our device could likewise be employed to explore the contributions of attentional and intentional factors in patients with neglect of vertical and radial space. ${ }^{17-20} \mathrm{We}$ manipulated the vertical dimension with the first patient, who had pronounced sensoryattentional neglect in both line bisection and cancellation tasks. Turning the monitor $90^{\circ}$ in the coronal plane produced the image of a vertically oriented line in perceptual space, although the line remained horizontally oriented in workspace. Under this condition, the patient's bisection error returned to the normal range. We also found at least one patient who omitted targets in both near space and left space in the direct condition (fig 2), a finding reported previously by others. ${ }^{21}$ In the indirect condition, the patients reversed their performance in both horizontal and near-far dimensions, thereby omitting targets in far-space and right-space. Although intriguing, our apparatus confounds the radial plane of the table top with the vertical plane of the monitor, rendering conclusion of attentional-intentional factors in radial or vertical space ambiguous. However, the device might be applied successfully to teasing apart the frames of reference involved in visuospatial processing along the radial and vertical axes. ${ }^{22}{ }^{23}$ Unlike previous methods, the body centred radial reference frame could be pitted against the environment centred vertical reference frame with a simple manipulation of camera angle rather than unwidely manipulation of the subject's body position.

Our a priori hypothesis was that the type of neglect (sensory-attentional, motor-intentional, or a combination of both) should be the same within a patient, regardless of the type of task. Our results did not support this hypothesis. Among nine patients in whom an attentional or intentional classification was possible, only six exhibited the same form of neglect with line bisection as they did with cancellation (two A-A and four I-I). Another three patients who showed motor-intentional neglect on the line bisection task showed a predominance of sensory-attentional neglect using the cancellation task (I-A group). Although we did not find any patients with sensory-attentional neglect on the line bisection and motor-intentional neglect on cancellation, our sample size was small. That three patients showed motor-intentional neglect in one task but sensory-attentional neglect in another suggests that their neglect may have been induced by a combination of defects. Furthermore, they might have shown different forms of neglect on these tasks because one task was more sensitive to intentional deficits (line bisection) and the other to attentional deficits (cancellation).

The findings of Binder et al consider the issue of differential processes involved in line bisection and cancellation. ${ }^{8}$ Studying patients after right hemispheric stroke, they found a group of patients showing pronounced bisection error but variable cancellation bias, a pattern strongly correlated with posterior brain injury. As these patients also performed poorly on a non-motor line judgment task, they asserted that rightward bisection shift resulted from impairment of perceptual mechanisms. By contrast, a second group with normal line bisection and abnormal cancellation performed normally on the line judgment task. Brain scans in these patients consistently found lesions in the frontal or basal ganglia region, implying a defect of the premotor contribution to neglect. Given their relatively small rightward deviation on direct line bisection, the I-A patients in our study may be most comparble to the second group of Binder et al. However, if injury to frontal/subcortical regions resulted simply in a directional hypokinesia or hypometria, then the performance of the I-A group presents a paradox: the preference to remain in right workspace during indirect bisection should have been exaggerated in the indirect cancellation task.

The reason why some patients are more likely to show motor-intentional neglect on line bisection and sensory-attentional neglect on cancellation is not clear. Reports of double dissociation between line bisection and cancellation performance in neglect hint at fundamental differences in the tasks' respective demands on selective and sustained attention. ${ }^{2}$ Although a detailed discussion of such differences extends beyond the scope of the present paper, consideration of certain aspects may illuminate the attentional-intentional discrepancy between tasks. During execution of either line bisection or cancellation, patients must attend to both sides of space and act in or toward the contralesional space. However, evidence from eye tracking experiments suggest that patients with neglect may make line length judgments on the basis of information obtained from a single visual fixation. ${ }^{24}$ By contrast, to target a cancellation task requires selective or focused attention (to discriminate targets from foils and previously cancelled targets ${ }^{25}$ ) as well as repeated redistribution of attention (disengagement, shift, and engagement). ${ }^{26}$ Furthermore, 
Chatterjee et al showed how neglect behaviour, as determined by cancellation, may be characterised by a limited capacity to distribute attention across time. ${ }^{27}$ Although admittedly speculative, the processing demands of line bisection may not exceed even a limited capacity system. Accordingly, the cancellation task may simply tax attentional resources more than does the line bisection task.

Alternatively, the indirect condition might confound the intentional aspect of eye/head movement during visual search with the intentional aspect of limb movement. In other words, if some patients failed to direct their eyes contralesionally but were able to move their limbs in this direction, they would show "attentional" neglect according to our definition. Similar reservations pertain to previously reported methods of dissociating attentional and intentional neglect. ${ }^{5-9}$ Unfortunately, the earlier studies were not able to compare line bisection versus cancellation performance. Despite this technical limitation apprehension of the entire array of lines or the entire bisection line on the monitor did not neccesitate movement of the head or eyes in patients with intact visual fields. Thus although separate intentional systems for eye and hand may be simultaneously deployed in the terminal guidance of either cancellation or bisection, we think that the distinction between sensoryattentional and motor-intentional subtypes retains validity as a first level approximation.

Perhaps the frontal system's "intentional" contribution to spatial cognition extends beyond biasing the direction and extent of eye and limb movement. At some level, frontal systems may also interact with posteriorattentional systems to construct and cohere environmental representations: "cognitive intention" may, for example, be required to disengage and redirect sensory transducers, shift attention from global to local domains, and update representations regarding the motivational value of stimuli. The reciprocal anatomical interconnections between components of the system mediating intention with that mediating attention certainly supports such interactions. ${ }^{28}$ For line bisection, the simultaneous perceptual comparison between right and left halves of a line might not recruit or induct much of this "cognitive intention". Target cancellation, by contrast, might entail conversion of global apprehension of the array to focal engagement of a single target, disengagement from the target after marking it, then shifting attention to an adjacent target while monitoring progress toward completion of the goal. Accordingly, when confronted with the bisection task, the I-A group's performance might have reflected primarily the motor aspects of intentional deficit, resulting in a tendency to remain on the right side of midline. When cancelling targets, on the other hand, the cognitive elements of the intentional defect supervened, causing degradation of perceptual processes and biasing attention away from contralateral perceptual space. Although admittedly speculative, our findings suggest such a complex interaction between the demands of a specific task and the residual capacities of a damaged neurocognitive network.

Another explanation for our results may be attentional "capture" of the rightward portion of the stimulus. Mark et $a l^{29}$ compared erasing versus marking targets from a cancellation array. Some patients showed a striking contrast between marking and erasing targets. Such patients showed left hemispatial neglect in the first condition but little or no neglect in the second condition, suggesting that neglect might be due to attentional capture from rightward targets rather than inadequate attention towards the left side of the array. This capture might exert different effects on line bisection than on cancellation as, as discussed earlier, the cancellation task may demand more spatially focused attention. If so, the intentional bias found during line bisection may be overcome on the cancellation task by the attraction of multiple targets on the right side of the monitor. In the indirect condition, then, even if the patient preferred to remain on the right side of the workspace, targets on the right side of perceptual space might "capture" attention so strongly that they would impel the patient's hand toward the left workspace. However, the presumption that a stimulus array would promote attentional capture more than a line remains unproved.

Whatever factors are responsible for discrepancy between tasks, closer scrutiny of the data also suggests that performance, even in the A-A and I-I group, reflects more than the simple pitting of attentional factors against intentional factors. Specifically, our patients with neglect tended to err more, with greater magnitude of bisection deviation and a larger number of omissions, in the indirect condition compared with the direct condition. Previous investigators have noted a similar phenomenon. For example, Tegner and Levander ${ }^{8}$ described patients with normal direct cancellation performance in whom the indirect condition unmasked latent lateralised omissions. Similarly, Bisiach et al reported that two of 15 patients showed significantly greater rightward deviation in the non-congruent condition compared with the congruent condition. Neither group of investigators speculated about the basis for such behaviour. Although we are likewise uncertain about the nature of this tendency, some clues might be provided through a more complex analysis of bisection error. For example, using the apparatus described herein, Schwartz et $a b^{30}$ recently considered differences in bisection error between the direct condition and indirect condition in normal subjects. The difference was considered both in terms of a primary bias defined by the error direction (switching from right to left, left to right versus staying on the same side of midline) and also a secondary bias defined by error magnitude (absolute error increased (discordant bias), decreased (concordant bias), or stayed the same). The only patient with concordant secondary bias in our small series showed attentional primary bias. Although the relevance of secondary bias is unclear, we are currently examining the relation between primary 
and secondary bias with a larger series of patients with neglect.

With respect to the neuroanatomical relations, some preliminary evidence suggests that anterior lesions are associated with motorintentional neglect and posterior lesions cause sensory-attentional neglect. ${ }^{5-8}$ As we found only a few cases with predominantly one form of neglect (two A-A and four I-I), our data cannot provide generalisable clinicoanatomical information. However, the findings were consistent with previous studies: the two A-A patients had primarily posterior lesions whereas three of four I-I patients had either predominantly anterior or subcortical injury. Furthermore, if we combine our data with the previous studies dissociating motor-intentional from sensory-attentional neglect (table 3), a more consistent relation emerges. Specifically, those patients with involvement of frontal brain structures or exclusively subcortical structures more commonly show motor-intentional neglect. By contrast, patients with brain injury outside the frontal region exclusively show sensory-attentional neglect. Using binomial expansion with an a priori likelihood of 0.5 , the association of sensory-attentional neglect with lesions outside the frontal region is significant $(p=0.00001)$. The relation of motorintentional neglect to frontal brain injury is also significant $(p=0.00037)$. Whereas this aggregate analysis seems to support an anatomicalfunctional association, studies with larger groups of patients may strengthen the relationship between functionally separable forms of neglect and their respective anatomic substrate. This information, in turn, might advance and promote the formulation of a rational, theory-based nosology of disorders of spatial cognition. This work was supported by the Medical Research Service of
the Department of Veterans' Affairs and the State of Florida Department of Elder Affairs.

After submission of this manuscript for review, an article was After submission of this manuscript for review, an article was
published that uses an epidiascope to separate perceptual from published that uses an epidiascope to separate perceptual from
premotor factors in patients with unilateral neglect (Nico D. premotor factors in patients with unilateral neglect (Nico D. Detecting directional hypokinesia:
Neuropsychologia 1996;34:471-4).

1 Heilman KM, Valenstein E, Watson RT. Neglect and related disorders. In: Heilman KM, Valenstein E, eds. Clinical neurospsychology. New York: Oxford University Press, 1993:243-93.
2 Halligan PW, Marshall JC. Left visuo-spatial neglect: a meaningless entity? Cortex 1992;28:525-35.

3 Mesulam M. A cortical network for directed attention and unilateral neglect. Ann Neurol 1981;10:309-25.

4 Bisiach E, Luzzatti C. Unilateral neglect of representational space. Cortex 1978;14:129-33.

5 Coslett HB, Bowers D, Fitzpatrick E, et al. Directional hypokinesia and hemispatial inattention in neglect. Brain 1990;113:475-86.

6 Halligan PW, Marshall JC. Perceptual cuing and perceptual motor compatability in visuospatial neglect: a single case study. Cognitive Neuropsychology 1989;6:423-35.

7 Bisiach E, Geminiani G, Berti A, et al. Perceptual and premotor factors of unilateral neglect. Neurology 1990;40 1278-81.

8 Tegner R, Levander M. Through a looking glass: a new technique to demonstrate directional hypokinesia in unilattechnique to demonstrate directional hyp

9 Harvey M, Milner AD, Roberts RC. An investigation of hemispatial neglect using the landmark task. Brain Cogn 1995;27:59-78.

10 Daffner KR, Ahern GL, Weintraub S, et al. Dissociation neglect behavior following sequential strokes in the right hemisphere. Ann Neurol 1990;28:97-101.

11 Binder J, Marshall R, Lazar R, et al. Distinct syndromes of hemineglect. Arch Neurol 1992;49:1187-94.

12 Milner AD, Harvey M. Toward a taxonomy of spatial neglect. Neuropsychological Rehabilitation 1994;4:177-81.

13 Albert ML. A simple test of visual neglect. Neurology 1973; 23:658-64.

14 Damasio H, Damasio AR. Lesion analysis in neuropsychology. New York: Oxford University Press, 1989.

15 Ladavas E. The role of visual attention in neglect: a dissociation between peceptual and directional motor dissociation between peceptual and directional mo

16 Bisiach E, Luzzatti C, Perani D. Unilateral neglect, Bisiach E, Luzzatti C, Perani D. Unilateral neglect,
representational schema, and consciousness. Brain 1975; 102:609-18.

17 Rapcsak SZ, CR Cimino, KM Heilman. Altitudinal neglect. Neurology 1988;38:277-81.

18 Mennemeier M, E Wertman, KM Heilman. Neglect of near peripersonal space. Brain 1992;115:37-50.

19 Butter CM, J Evans, N Kirsch, et al. Altitudinal neglect following traumatic brain injury: a case report. Cortex 1989;25:135-46.

20 Shelton PA, D Bowers, KM Heilman. Peripersonal and vertical neglect. Brain 1990;113:191-205.

21 Halligan PW, Marshall JC. Is neglect (only) lateral? A quadrant analysis of line cancellation. F Clin Exp Neuropsychol 1989;11:793-8.

22 Mennemeier M, Chatteriee A, Heilman KM. A comparison of the influences of body and environment centered of the influences of body and environment centerec

23 Adair JC, Williamson DJ, Jacobs DH, et al. Neglect of the radial and vertical space: importance of the retinotopic ref-
erence frame. F Neurol Neurosurg Psychiatry 1995;58:724-8.

24 Ishiai S, Furukawa T, Tsukagoshi H. Visuospatial processes of line bisection and the mechanisms underlying unilateral spatial neglect. Brain 1989;112:1485-502.

25 Rapcsak SZ, Fleet WS, Verfaellie M, et al. Selective attention in hemispatial neglect. Arch Neurol 1989;46:178-82.

26 Posner MI, Walker J, Friedrich FJ, et al. Effects of parietal lobe injury on covert orienting of visual attention. $\mathcal{f}$ Neurosci $1984 ; 4: 163-87$.

27 Chatterjee A, Mennemeier M, Heilman KM. Search patterns and neglect: a case study. Neuropsychologia 1992;30:657-72.

28 Pandya DM, Kuypers HGJM. Cortico-cortical connections in the rhesus monkey. Brain Res 1969;13:13-36. 29 Mark V, Kooistra C, Heilman KM. Hemispatial neglect 1207-11.

30 Schwartz RL, Adair JC, Na D, et al. Spatial bias: attentional and intentional influences in normal subjects. Neurology 1997;48:234-49. 